\title{
Noncoherent Sequence Detection of Continuous Phase Modulations
}

\author{
Giulio Colavolpe, Student Member, IEEE, and Riccardo Raheli, Member, IEEE
}

\begin{abstract}
In this paper, noncoherent sequence detection, proposed in a companion paper [1] by Colavolpe and Raheli, is extended to the case of continuous phase modulations (CPM's). The results in the companion paper on linear modulations with intersymbol interference (ISI) are used here because a CPM signal is mathematically equivalent to a sum of ISI-affected linearly modulated components, according to the Laurent decomposition.

The proposed suboptimal detection schemes have a performance which approaches that of coherent detection with acceptable complexity, allow for time-varying phase models, and compare favorably with previously proposed solutions.
\end{abstract}

Index Terms - Continuous phase modulation, intersymbol interference, maximum-likelihood detection, noncoherent sequence detection.

\section{INTRODUCTION}

$\mathbf{N}$ ONCOHERENT detection of digital signals is an attractive strategy in situations where carrier phase recovery is difficult because most of the drawbacks of a phase-locked loop (PLL), used to approximately implement coherent detection, may be avoided. Specifically, typical problems of PLL's, such as false-locks, phase slips, or losses of lock caused by severe fading or oscillator frequency instabilities, are simply by-passed (see [1, refs. [2]-[5]]).

The simplest noncoherent receivers for continuous phase modulation (CPM) are differential detectors [2]. A different approach to differential detection is presented in [3], based on Laurent decomposition of CPM signals as a sum of linearly modulated components [4]. In [3], the author approximates the branch metrics of a coherent receiver estimating the carrier phase, on the basis of the previous observation, under the maximum-likelihood (ML) criterion. The obtained receiver uses a Viterbi algorithm (VA); nevertheless, its performance is far from that of a coherent detector because of the differential detection approach.

The performance of coherent detection may be approached by more complex noncoherent receivers based on multiplesymbol differential detection. This approach, first presented for linear modulations (see references in [1]), was extended to minimum shift keying (MSK) [5], full-response CPM [6], and Gaussian MSK (GMSK) [7]. Multiple-symbol dif-

Paper approved by E. Eleftheriou, the Editor for Equalization and Coding of the IEEE Communications Society. Manuscript received September 15, 1997; revised September 30, 1998 and February 3, 1999. This work was performed within a research cooperation between Dipartimento di Ingegneria dell'Informazione, Università di Parma, Italy and Italtel S.p.A., Milano, Italy. This paper was presented in part at the International Symposium on Information Theory (ISIT '98), Cambridge, MA, USA, August 1998, and the Global Communications Conference (GLOBECOM '98), Sydney, Australia, November 1998.

The authors are with the Dipartimento di Ingegneria dell'Informazione, Università di Parma, 43100 Parma, Italy.

Publisher Item Identifier S 0090-6778(99)06289-3. ferential receivers are based on ML detection of a block of information symbols, based on a finite-duration signal observation. A different approach to noncoherent detection, based on a limited tree-search algorithm, is proposed in [8]. A reduced-complexity multiple differential detection algorithm for CPM schemes, which makes use of the output of $j$-symbol differential detectors processed in an optimal manner using a VA, is presented in [9]. Another trellis-based noncoherent detection scheme is considered in [10]. In general terms, the performance of noncoherent detection schemes based on extended observation windows improves, for increasing observation length and receiver complexity, and approaches that of optimal coherent detection. This result was first noted for CPM in [11] and confirmed in most of the cited references.

In this paper, we extend the noncoherent sequence detection algorithms proposed in [1] to CPM's. The extension is based on Laurent decomposition, recently extended to multilevel signaling [4], which mathematically describes a CPM signal as a sum of linearly modulated components affected by ISI. The results in [1] on linear modulations with ISI are extended to CPM using a multidimensional whitening filter (WF). An alternative solution that does not need a multidimensional WF was presented in [12].

\section{Noncoherent SEQUENCE Detection of CPM}

The complex envelope of CPM signals has the form [2]

$$
s(t, \boldsymbol{a})=\sqrt{\frac{2 E_{S}}{T}} \exp \left\{j 2 \pi h \sum_{n} a_{n} q(t-n T)\right\}
$$

in which $E_{S}$ is the energy per information symbol, $T$ is the symbol interval, $h=k / p$ is the modulation index ( $k$ and $p$ are relatively prime integers), the information symbols $\left\{a_{n}\right\}$ are assumed independent and take on values in the $M$-ary alphabet $\{ \pm 1, \pm 3, \cdots, \pm(M-1)\}$ with equal probability, and the vector $\boldsymbol{a}$ denotes the information sequence. The function $q(t)$ is the phase-smoothing response and its derivative $g(t)$ is the frequency pulse, assumed of duration $L_{g} T$. An extension to the use of channel coding techniques may be dealt with by the methods described for coded linear modulations in [1].

Based on Laurent representation, the complex envelope of CPM signals may be exactly expressed as [4]

$$
s(t, \boldsymbol{a})=\sum_{k=0}^{Q^{\log _{2} M}(M-1)-1} \sum_{n} \alpha_{k, n} h_{k}(t-n T)
$$

in which $M$ is assumed to be a power of two to simplify the notation, $Q \triangleq 2^{L_{g}-1}$, and the expressions of pulses $\left\{h_{k}(t)\right\}$ and symbols $\left\{\alpha_{k, n}\right\}$ as a function of the information symbol 
sequence $\left\{a_{n}\right\}$ may be found in [4] (see this reference for the general case of $M$ nonpower of two). It is known that the output, sampled at the symbol rate, of a bank of filters, matched to the pulses $h_{k}(t)$ is a set of sufficient statistics for coherent detection of a CPM signal (e.g., see [13]). Proceeding as in [1], it may be shown easily that this is also true for noncoherent sequence detection.

By truncating the summation in (2) considering only the first $K<Q^{\log _{2} M}(M-1)$ terms, we obtain an approximation of $s(t, \boldsymbol{a})$. Most of the signal power is concentrated in the first $M-1$ components, i.e., those associated with the pulses $\left\{h_{k}(t)\right\}$ with $0 \leq k \leq M-2$, which are denoted as principal pulses [4]. As a consequence, a value of $K=M-1$ may be used in (2) to attain a very good tradeoff between approximation quality and number of signal components. In this case, the approximation may be slightly improved by modifying the pulses $\left\{h_{k}(t)\right\}$ in order to minimize the mean square error with respect to the exact signal [4]. In [13], it was shown that a coherent receiver based only on principal pulses practically attains the performance of an optimal coherent detector.

As in [1], the complex envelope of the received signal may be expressed as

$$
r(t)=s(t, \boldsymbol{a}) e^{j \theta}+w(t)
$$

where the phase rotation $\theta$ is modeled as a random variable with uniform distribution in the interval $[0,2 \pi)$ and $w(t)$ is a complex-valued Gaussian white noise process with independent components, each with two-sided power spectral density $N_{0}$. We now introduce suboptimal noncoherent detection schemes for CPM signals. We consider a simplified representation, based on principal pulses only, which allows us a significant complexity reduction with negligible performance loss. The output, sampled at time $n T$, of a filter matched to pulse $h_{k}(t)$ may be expressed as

$$
\left.x_{k, n} \triangleq r(t) \otimes h_{k}(-t)\right|_{t=n T}=s_{k, n} e^{j \theta}+n_{k, n}
$$

where

$$
\begin{aligned}
n_{k, n} & \left.\triangleq w(t) \otimes h_{k}(-t)\right|_{t=n T} \\
s_{k, n} & \left.\triangleq s(t, \boldsymbol{a}) \otimes h_{k}(-t)\right|_{t=n T} \\
& =\sum_{m=0}^{K-1} \sum_{i} \alpha_{m, i} g_{m, k}[(n-i) T]
\end{aligned}
$$

and

$$
g_{m, k}(t) \triangleq h_{m}(t) \otimes h_{k}(-t)
$$

From (6), we may easily observe that $s_{k, n} \neq \alpha_{k, n}$ because of ISI and interference from other signal components. Regarding the noise terms, they are characterized by the following crosscorrelation function

$$
E\left\{n_{m}(t) n_{k}^{*}(t-\tau)\right\}=2 N_{0} g_{m, k}(-\tau)
$$

which depends on the shape of pulses $g_{m, k}(t)$. In order to obtain a suboptimal noncoherent receiver with good performance and affordable state-complexity, it is convenient to transform this set of sufficient statistics in an alternative one by means of a whitening procedure [1].

It is convenient to define

$$
\begin{aligned}
& \boldsymbol{x}_{n} \triangleq\left(x_{0, n}, x_{1, n}, \cdots, x_{K-1, n}\right)^{T} \\
& \boldsymbol{s}_{n} \triangleq\left(s_{0, n}, s_{1, n}, \cdots, s_{K-1, n}\right)^{T} \\
& \boldsymbol{n}_{n} \triangleq\left(n_{0, n}, n_{1, n}, \cdots, n_{K-1, n}\right)^{T} \\
& \boldsymbol{\alpha}_{n} \triangleq\left(\alpha_{0, n}, \alpha_{1, n}, \cdots, \alpha_{K-1, n}\right)^{T} \\
& \boldsymbol{G}_{n} \triangleq\left[g_{i, j}(n T)\right], \quad i, j=0,1, \cdots, K-1
\end{aligned}
$$

which represent, in matrix notation, the output of the bank of $K$ matched filters, its signal and noise components, the symbols of the $K$ linearly modulated signal components [see (2)], and the $K \times K$ overall matrix impulse response at the output of the matched filter bank, respectively, all at discrete time $n$. With this matrix notation, the observation vector may be expressed as

$$
\boldsymbol{x}_{n}=\boldsymbol{s}_{n} e^{j \theta}+\boldsymbol{n}_{n}=e^{j \theta} \sum_{l=-L}^{L} \boldsymbol{G}_{l}^{T} \boldsymbol{\alpha}_{n-l}+\boldsymbol{n}_{n}
$$

where $L$ is related to the duration $L_{g}$ of the frequency pulse and plays the role of a modulation memory parameter. The matrix covariance sequence of the discrete vector noise process may also be defined as

$$
\boldsymbol{R}_{\boldsymbol{n}}(m) \triangleq E\left\{\boldsymbol{n}_{n} \boldsymbol{n}_{n-m}^{* T}\right\}=2 N_{0} \boldsymbol{G}_{-m}=2 N_{0} \boldsymbol{G}_{m}^{T}
$$

where the property $g_{m, k}(t)=g_{k, m}(-t)$ has been used.

The following bilateral $Z$-transforms of the previously introduced matrix sequences may be defined:

$$
\begin{aligned}
& \boldsymbol{X}(z) \triangleq \sum_{m=-\infty}^{\infty} \boldsymbol{x}_{m} z^{-m} \\
& N(z) \triangleq \sum_{m=-\infty}^{\infty} \boldsymbol{n}_{m} z^{-m} \\
& \boldsymbol{A}(z) \triangleq \sum_{m=-\infty}^{\infty} \boldsymbol{\alpha}_{m} z^{-m} \\
& \boldsymbol{G}(z) \triangleq \sum_{m=-\infty}^{\infty} \boldsymbol{G}_{m} z^{-m} \\
& \boldsymbol{\Phi}_{\boldsymbol{n}}(z) \triangleq \sum_{m=-\infty}^{\infty} \boldsymbol{R}_{\boldsymbol{n}}(m) z^{-m}=2 N_{0} \boldsymbol{G}\left(z^{-1}\right)=2 N_{0} \boldsymbol{G}^{T}(z) .
\end{aligned}
$$

The spectral matrix $\boldsymbol{\Phi}_{\boldsymbol{n}}(z)$ of the vector process $\boldsymbol{n}_{n}$ is assumed to be positive definite along the unit circle (by definition, it is nonnegative definite) [14]. If the determinant $\left|\boldsymbol{\Phi}_{\boldsymbol{n}}(z)\right|$ is identically equal to zero on the unit circle, it is straightforward to show that the discrete processes $\left\{n_{k, n}\right\}$ are linearly depen- 
dent. In this case, an alternative set of sufficient statistics is simply obtained discarding the sampled outputs $\left\{x_{k, n}\right\}$ whose noise components can be expressed as a linear combination of the others (with probability one). This condition is never verified for CPM (in a large number of considered cases). However, in certain cases $\boldsymbol{\Phi}_{\boldsymbol{n}}(z)$ could be ill-conditioned. In this case, a simple countermeasure is to discard some signal components. For example, for the quaternary 2RC (raisedcosine frequency pulse with $L_{g}=2$ ) scheme considered in the numerical results, two principal pulses, namely $h_{1}(t)$ and $h_{2}(t)$, are quite similar. These two pulses can be replaced by an average equivalent one, $h_{e}(t)$, with corresponding symbol $\alpha_{e, k} \triangleq \alpha_{1, k}+\alpha_{2, k}$.

Under the above positive definiteness assumption for the spectral matrix $\boldsymbol{\Phi}_{\boldsymbol{n}}(z)$ along the unit circle, it is possible to find a matrix $\boldsymbol{F}(z)$ [14], [15] such that

$$
\boldsymbol{\Phi}_{\boldsymbol{n}}(z)=2 N_{0} \boldsymbol{G}^{T}(z)=2 N_{0} \boldsymbol{F}\left(z^{-1}\right) \boldsymbol{F}^{T}(z)
$$

and such that the determinant $\left|\boldsymbol{F}\left(z^{-1}\right)\right|$ has no roots inside the unit circle. Therefore, an alternative set of sufficient statistics is obtained by filtering the multidimensional signal $\left\{\boldsymbol{x}_{n}\right\}$ with a multidimensional $K \times K$ filter whose transfer function is $\boldsymbol{F}^{-1}\left(z^{-1}\right)$. The resulting vector signal may be expressed as

$$
z_{n}=\boldsymbol{y}_{n} e^{j \theta}+\boldsymbol{w}_{n}
$$

where $\left\{\boldsymbol{y}_{n}\right\}$ is the result of an identical filtering on the signal component $\left\{\boldsymbol{s}_{n}\right\}$.

The $Z$-transform of (14) is

$$
\begin{aligned}
\boldsymbol{X}(z) & =\boldsymbol{G}^{T}(z) \boldsymbol{A}(z) e^{j \theta}+\boldsymbol{N}(z) \\
& =\boldsymbol{F}\left(z^{-1}\right) \boldsymbol{F}^{T}(z) \boldsymbol{A}(z) e^{j \theta}+N(z) .
\end{aligned}
$$

Hence, the $Z$-transform of $\left\{\boldsymbol{y}_{n}\right\}$ is

$$
\boldsymbol{Y}(z)=\sum_{m=-\infty}^{\infty} \boldsymbol{y}_{m} z^{-m}=\boldsymbol{F}^{T}(z) \boldsymbol{A}(z)
$$

It is straightforward to show that the inverse transform of $\boldsymbol{F}(z)$ has only $L+1$ nonzero element $\boldsymbol{F}_{\boldsymbol{l}}$. As a consequence, signal $\left\{\boldsymbol{y}_{n}\right\}$ may be expressed as

$$
\boldsymbol{y}_{n}=\sum_{l=0}^{L} \boldsymbol{F}_{l}^{T} \boldsymbol{\alpha}_{n-l}
$$

As the spectral matrix of the vector process $\boldsymbol{w}_{n}$ is

$$
\boldsymbol{\Phi}_{\boldsymbol{w}}(z)=\boldsymbol{F}^{-1}\left(z^{-1}\right) \boldsymbol{\Phi}_{\boldsymbol{n}}(z) \boldsymbol{F}^{-1 T}(z)=2 N_{0} \boldsymbol{I}
$$

where $\mathbf{I}$ is a $K \times K$ identity matrix, the filter $\boldsymbol{F}^{-1}\left(z^{-1}\right)$ is a multidimensional WF which results from the above generalization of the WF used in [16]. A physical realization of this filter requires a delay to assure causality.

This multidimensional receiver front-end may be interpreted as a one-input $K$-output whitened matched filter (WMF) in the sense of [16], realized as the cascade of a one-input $K$-output matched filter followed by a $K \times K \mathrm{WF}$. In this derivation, we have not considered the case of a determinant of the spectral matrix $\boldsymbol{\Phi}_{\boldsymbol{n}}(z)$ with zeros along the unit circle because, in our experience, this is not a case of practical relevance in CPM schemes. However, this situation may be approached by generalizing the concept of pole-zero cancellation used in [16] to define the WMF in the case of signals with spectral nulls.

Using this set $\left\{\boldsymbol{z}_{n}\right\}$ of sufficient statistics, the optimal noncoherent sequence detection strategy may be derived as a straightforward extension of the strategy in [1] for ISIaffected linear modulations, in which a summation over the $K$ components of the CPM signal is present. Proceeding with the approximations described in [1], the incremental or branch metrics read

$$
\begin{aligned}
\lambda_{n}(\tilde{\boldsymbol{a}}) \triangleq & \left|\sum_{k=0}^{K-1} \sum_{i=0}^{N-1} z_{k, n-i} \tilde{y}_{k, n-i}^{*}\right| \\
& -\left|\sum_{k=0}^{K-1} \sum_{i=1}^{N-1} z_{k, n-i} \tilde{y}_{k, n-i}^{*}\right|-\frac{1}{2} \sum_{k=0}^{K-1}\left|\tilde{y}_{k, n}\right|^{2}
\end{aligned}
$$

where $y_{k, n}$ is the $k$ th element of vector $\boldsymbol{y}_{n}$ in (25) and $\tilde{y}_{k, n}$ denote versions of $y_{k, n}$ corresponding to each hypothetical information sequence. The number of states depends on the parameter $N$. For example, using principal pulses only, i.e., a value of $K=M-1$, for which $\boldsymbol{\alpha}_{n}$ depends only on $a_{n}$ (see [13]), we have a number of trellis states equal to $S=M^{N+L-1}$. This complexity may be limited by a possible use of techniques for state-complexity reduction, described in [1], in order to limit the number of states without excessively reducing the value of $N$. As in [1], even using small values of $N$ (a few units), a performance close to that of coherent detection may be obtained. The baseband equivalent model of the receiver is shown in Fig. 1.

An alternative approach to noncoherent sequence detection of CPM that does not need a multidimensional WMF is described in [12]. This approach was first used for binary CPM in [3] to derive differential detectors which take into account the inherent ISI of the linearly modulated signal components and the correlation of noise samples at the output of the matched filters. In [12], a generalization to multilevel CPM and receivers with an implicit phase memory $N>2$ is proposed.

\section{NUMERICAL RESULTS}

In this section, we assess the performance of the proposed noncoherent sequence detection schemes by means of computer simulations, in terms of bit-error rate (BER) versus $E_{b} / N_{0}, E_{b}$ being the received signal energy per information bit.

As examples of binary CPM with $h=0.5$, in Fig. 2, we consider GMSK with parameter $B T=0.25$ [2]. The proposed receiver, based on the branch metrics (27), takes only into account the first signal component with pulse $h_{0}(t)$, i.e., $K=$ 1. This choice corresponds to an approximation of the GMSK signal as a linear modulation (hence, the branch metrics are 


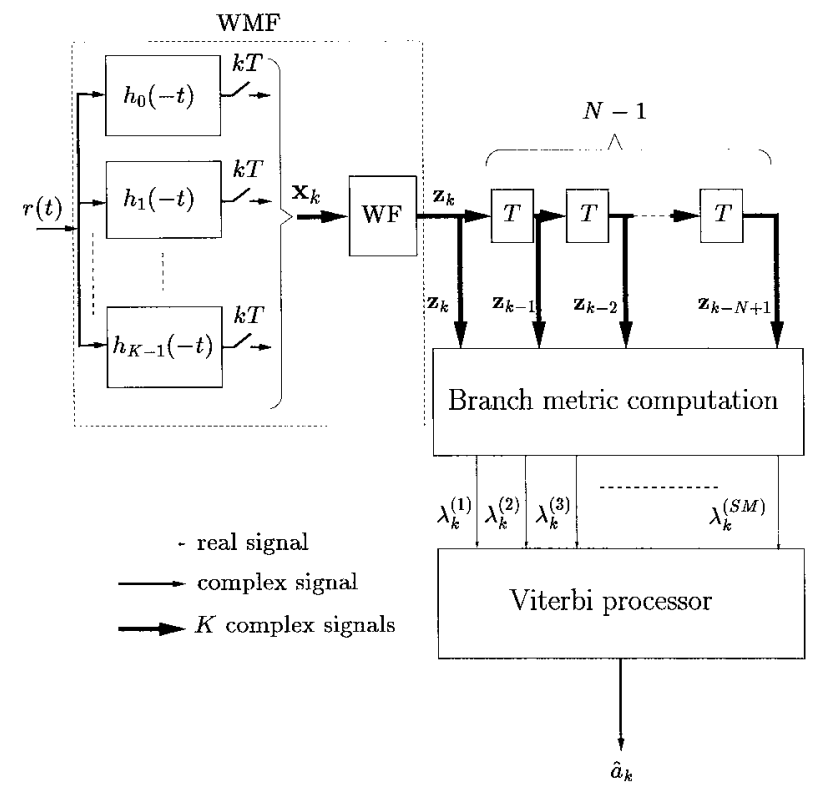

Fig. 1. Noncoherent sequence detection receiver for CPM.

equivalent to those in [1]). The receiver front-end is a standard WMF. Several levels of complexity are considered by selecting different values of implicit phase memory $N$ and number of states $S$. Most of them use complexity-reduction techniques, as described in [1]. Since the pulse $g_{0,0}(t)$ is characterized by five significant samples only, the modulation memory parameter is $L=2$. The performance of the optimal coherent receiver is also shown for comparison. ${ }^{1}$ The performance approaches that of coherent detection for increasing levels of complexity. State-complexity reduction is an efficient tool because in most cases it entails negligible degradation. Overall, a loss inferior to $1.5 \mathrm{~dB}$ may be achieved by receivers which search four- or eight-state trellis diagrams.

As an example of multilevel CPM, we consider a quaternary raised-cosine (RC) modulation [2] with frequency pulse of duration $L_{g}=2$ symbol intervals (2RC) and modulation index $h=0.25$. As noted in Section II, due to the similarity of two of the three principal pulses, we may substitute the corresponding matched filters with an average one. Therefore, we consider receivers with two matched filters, a two-dimensional WF (in this case, $L=1$ ) and a VA with branch metrics (27). Fig. 3 shows the performance of these receivers along with that of a coherent receiver and the noncoherent receiver in [12]. The results previously described for binary CPM are confirmed for this quaternary scheme. For increasing complexity levels, the receiver performance approaches that of coherent detection-a loss of only $1 \mathrm{~dB}$ may be attained with affordable complexity levels. For limited complexity, the proposed receiver performs better than the receiver in [12].

\section{CONCLUSIONS}

In this paper, noncoherent sequence detection, proposed in [1], is extended to CPM on the basis of Laurent representation.

\footnotetext{
${ }^{1} \mathrm{~A}$ linear approximation of the modulation would allow us to use a receiver based on decision-feedback equalization. Compared to optimal detection, this receiver exhibits a performance loss of only about $0.2 \mathrm{~dB}$ [13].
}

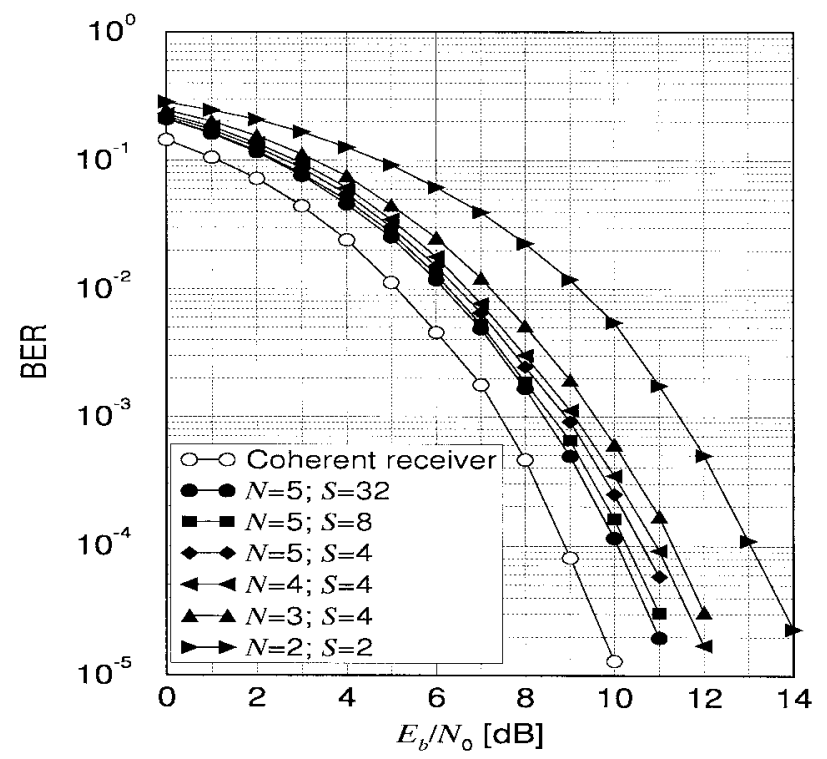

Fig. 2. BER of the proposed detection schemes for GMSK with $B T=0.25$ for different values of implicit phase memory $N$ and number of states $S$.

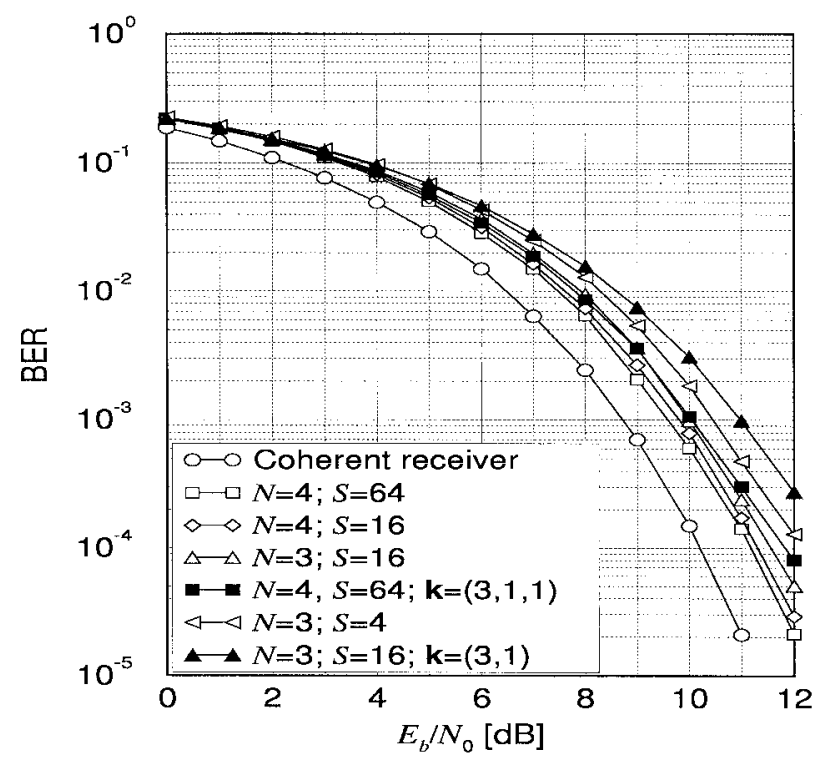

Fig. 3. BER of the proposed detection schemes for quaternary $2 \mathrm{RC}$ modulation with $h=0.25$ for different values of implicit phase memory $N$ and number of states $S$ (white marks). The performance of the receivers in [12] is also shown (black marks).

As in the case of coherent detection, the sampled output of a bank of matched filters is a sufficient statistic for noncoherent sequence detection. By a multidimensional WF, a one-input multi-output WMF may be defined, which provides an alternative set of sufficient statistics. Although equivalent to the matched filter bank output for ideal noncoherent sequence detection, the latter set of sufficient statistics is significantly more efficient for the proposed suboptimal detection schemes because it allows truncation of the (theoretically unlimited) memory of proper incremental metrics to levels that entail affordable receiver complexity.

The performance of the proposed detection schemes has been assessed by computer simulation for binary and quater- 
nary CPM. The tradeoff between performance and complexity may be controlled by the number of signal components, the implicit phase memory parameter, and the level of statecomplexity reduction. Being noncoherent, these schemes do not have the typical drawbacks of conventional approximation of coherent detection based on the use of PPL and are very robust to phase jitter and frequency offsets.

\section{REFERENCES}

[1] G. Colavolpe and R. Raheli, "Noncoherent sequence detection," IEEE Trans. Commun., this issue, pp. 1376-1385.

[2] J. B. Anderson, T. Aulin, and C.-E. Sundberg, Digital Phase Modulation. New York: Plenum, 1986.

[3] G. K. Kaleh, "Differential detection via the Viterbi algorithm for offset modulation and MSK-type signals," IEEE Trans. Veh. Technol., vol. 41, pp. 401-406, Nov. 1992.

[4] U. Mengali and M. Morelli, "Decomposition of $M$-ary CPM signals into PAM waveforms," IEEE Trans. Inform. Theory, vol. 41, pp. 1265-1275, Sept. 1995.

[5] H. Leib and S. Pasupathy, "Noncoherent block demodulation of MSK with inherent and enhanced encoding," IEEE Trans. Commun., vol. 40, pp. 1430-1441, Sept. 1992.

[6] M. K. Simon and D. Divsalar, "Maximum-likelihood block detection of noncoherent continuous phase modulation," IEEE Trans. Commun., vol. 41, pp. 90-98, Jan. 1993.
[7] A. Abrardo, G. Benelli, and G. Cau, "Multiple-symbol differential detection of GMSK for mobile communications," IEEE Trans. Veh. Technol., vol. 44, pp. 379-389, Aug. 1995.

[8] S. T. Andersson and N. A. B. Svensson, "Noncoherent detection of convolutionally encoded continuous phase modulation," IEEE J. Select. Areas Commun., vol. 7, pp. 1402-1414, Dec. 1989.

[9] D. Makrakis and K. Feher, "Multiple differential detection of continuous phase modulation signals," IEEE Trans. Veh. Technol., vol. 42, pp. 186-196, May 1993.

[10] D. Raphaeli, "Noncoherent coded modulation," IEEE Trans. Commun., vol. 44, pp. 172-183, Feb. 1996.

[11] T. Aulin and C.-E. Sundberg, "Partially coherent detection of digital full response continuous phase modulated signals," IEEE Trans. Commun., vol. COM-30, pp. 1096-1117, May 1982.

[12] G. Colavolpe and R. Raheli, "Noncoherent sequence detection of CPM," Electron. Lett., vol. 34, no. 3, pp. 259-261, Feb. 1998.

[13] CPM signals," IEEE Trans. Commun., vol. 45, pp. 1070-1079, Sept. 1997.

[14] P. R. Mothyka and J. A. Cadzow, "The factorization of discreteprocess spectral matrices," IEEE Trans. Automat. Contr., vol. AC-12, pp. 698-707, Dec. 1967

[15] D. N. Prabhakar Murthy, "Factorization of discrete-process spectral matrices," IEEE Trans. Inform. Theory, vol. IT-19, pp. 693-696, Sept. 1973.

[16] G. D. Forney Jr., "Maximum-likelihood sequence estimation of digital sequences in the presence of intersymbol interference," IEEE Inform. Theory, vol. IT-18, pp. 363-378, May 1972. 